\title{
Heavy ion beam measurement of the hydration of cementitious materials
}

\author{
R.A. Livingston $^{a}$, J.S. Schweitze ${ }^{b}$, C. Rolfs ${ }^{c}$, H.-W. Becker ${ }^{c}$, S. Kubsky ${ }^{d}$, T. Spillane ${ }^{b}$, J. Zickefoose ${ }^{b}$, \\ M. Castellote ${ }^{\mathrm{e}}$, P.G. de Viedma ${ }^{\mathrm{e}}$, J. Cheung ${ }^{\mathrm{f}}$ \\ ${ }^{+}$Materials Science \& Engineering Department, University of Maryiand, College Park, MD 20742, USA \\ 'University of Connecticut, Storrs, CT 06269-3046, USA \\ ' Ruirt-Universität Bocium, Bocium 0-44780, Gemony \\ 'Syncivotron SOLEIL, Saint-Aubin, 91192 Gif-sur-Vvette, CEDEX, France \\ "Institute of Construction Science "Eduardo Tonoja" (CSIC), Madidi 28080, Spain \\ 'W.R. Grace, Cambridge, MA 02140, USA
}

Keywords:

Portland cement

Nuclear resonance reaction analysis

Hydrogen depth profile

lnduction time

\begin{abstract}
A B S T R A T
The setting and development of strength of Portland cement concrete depends upon the reaction of water with various phases in the Portland cement. Nuclear resonance reaction analysis (NRRA) involving the ${ }^{1} \mathrm{H}\left({ }^{15} \mathrm{~N}, \alpha, \gamma\right)^{12} \mathrm{C}$ reaction has been applied to measure the hydrogen depth profile in the few $100 \mathrm{~nm}$ thick surface layer that controls the early stage of the reaction. Specific topics that have been investigated include the reactivity of individual cementitious phases and the effects of accelerators and retarders.
\end{abstract}

\section{Introduction}

The setting time is a critical factor in the technology of Portland cement concrete. However a complete scientific understanding of the process has not yet been achieved. The setting time is a macroscopic property measured by engineering tests; the equivalent on the nanometer scale is the induction time which is controlled by fundamental physical and chemical processes. It has been established that factors such as temperature and certain types of chemicals can modify the induction time, but several competing models have been proposed for the mechanisin that determines it (Taylor, 1997; Young, 2005).

It is inferred from mass balance considerations based on the amount of reaction products during this period, that a layer of only a few tens of nanometers on the surface of the cement grains must be involved (Taylor, 1997). The surface layer has been examined by several instiumental techniques (Henderson and Bailey, 1993; Menetrier et al., 1979; Meredith et al., 1995; Mitchell et al., 1996; Scrivener, 1987; Viehland et al., 1996), but these have yielded mostly qualitative results. We have applied a complementary technique, Nuclear resonance reaction analysis (NRRA), that provides in-situ measurements of the analytical hydrogen concentration as a function of depth with a resolution of a few nanometers (Livingston et al., 2001).

There are a number of nuclear resonance reactions that can be used for hydrogen detection (Lanford, 1995). The specific NRRA method used here is based on the $E_{\mathrm{R}}=6.400 \mathrm{MeV}$ resonance in the ${ }^{1} \mathrm{H}\left({ }^{15} \mathrm{~N}, \alpha, \gamma\right){ }^{12} \mathrm{C}$ reaction. Owing to the strong isolated resonance in the $\mathrm{H}$ capture cross section, the reaction only occurs when the ${ }^{15} \mathrm{~N}$ ion energy is at the resonance energy. If its energy is greater than the resonance, no reactions occur until it loses enough energy by atomic scattering to get down to the resonance energy. Thus the larger the difference between the beam energy and the resonance energy, the greater the depth penetration of the ${ }^{15} \mathrm{~N}$ ion before it reaches resonance capture.

\section{Experimental apparatus and methods}

The NRRA measurements are done at the $4 \mathrm{MeV}$ Dynamitron tandem accelerator at the Ruhr-Universität Bochum in Bochum, Germany. Typical particle currents at the target are about $15 \mathrm{nA}$. The $4.44 \mathrm{MeV} \gamma$-ray emitted in the ${ }^{1} \mathrm{H}\left({ }^{15} \mathrm{~N}, \alpha, \gamma\right){ }^{12} \mathrm{C}$ reaction is obseived with a 12 in. diameter $\times 12$ in. $\mathrm{Nal}(\mathrm{TI})$ crystal $(49 \pm 3 \%$ photopeak efficiency) connected to a 1024 channel multichanne] analyzer.

High resolution NRRA requires a beam of nitrogen jons with very precisely regulated energies. The Bochum facility uses a radio-frequency system, rather than a mechanical pelletron or belt, to generate the accelerating voltage with very low ripple and good energy resolution. Moreover, careful analysis and correction of all steps from production of the beam in the jon source through acceleration and transport onto the target has been carried out to minimize the variance in the beam energy (Borucki et al., 1999; Wüstenbecker et al., 1987). This degree of precision combined 
with the high stopping cross section for ${ }^{15} \mathrm{~N}$ beams in cementitious materials provides an H-detection sensitivity of about $10 \mathrm{ppm}$ and a $\mathrm{H}$-depth resolution of a few $\mathrm{nm}$ at the surface. This has enabled the investigation in detail of the effect of temperature and other factors on the induction period (Schweitzer et al., 2001; Schweitzer et al., 2003; Schweitzer et al., 2005).

The basic experimental procedure has been described elsewhere (Livingston et al., 2001). Cementitious phases such as tricalcium silicate $\left(C_{3} S\right)$ or tricalcium aluminate $\left(C_{3} A\right)$ were molded into cylindrical pellets of $12.7 \mathrm{~mm}$ diameter and then fused. A typical experimental run for determining the induction time for a particular condition consists of 8-12 samples, not including control samples. They are hydrated in a common bath of solution of specified composition and temperature, and individual samples are then removed at specific times for analysis. The samples are stored and handled under inert atmosphere both before and after the chemical reaction. A pure methanol rinse is used to remove any water on the sample. Reacted samples are then kept in a vacuum system until analysis. Each sample thus represents a single point in the material's hydration time history.

\subsection{Data acquisition}

The sample is mounted in a chamber at the end of a beam line, and the chamber is then evacuated to a pressure of $10^{-6}$ Torr. To probe the sample's $\mathrm{H}$ depth profile, the beam energy is increased stepwise starting just below the resonance energy of $6.400 \mathrm{MeV}$. At each energy step, a $\gamma$-ray spectrum is acquired, and the area under the $4.44 \mathrm{MeV}$ peak is calculated. By plotting this hydrogen signal as a function of incident beam energy it is possible to visualize the hydrogen depth profile.

An example of such a profile is shown in Fig. 1. This is for a $\mathrm{C}_{3} \mathrm{~S}$ sample during the induction period. It shows the typical Gaussian peak associated with a surface layer, on the left edge of the figure, followed by a diffusion-type region extending into the sample. The Gaussian peak is interpreted as a semi-permeable surface layer. This allows the calcium ions to migrate from the substrate into the solution in exchange for water ions, but the larger silicate ions are trapped below the surface in the form of a silica gel. A schematic of the various layers is shown in Fig. 2. The gel has a larger volume than the $\mathrm{C}_{3} \mathrm{~S}$, and as it grows it exerts stresses on the surface layer. Eventually the stress exceeds the strength of the

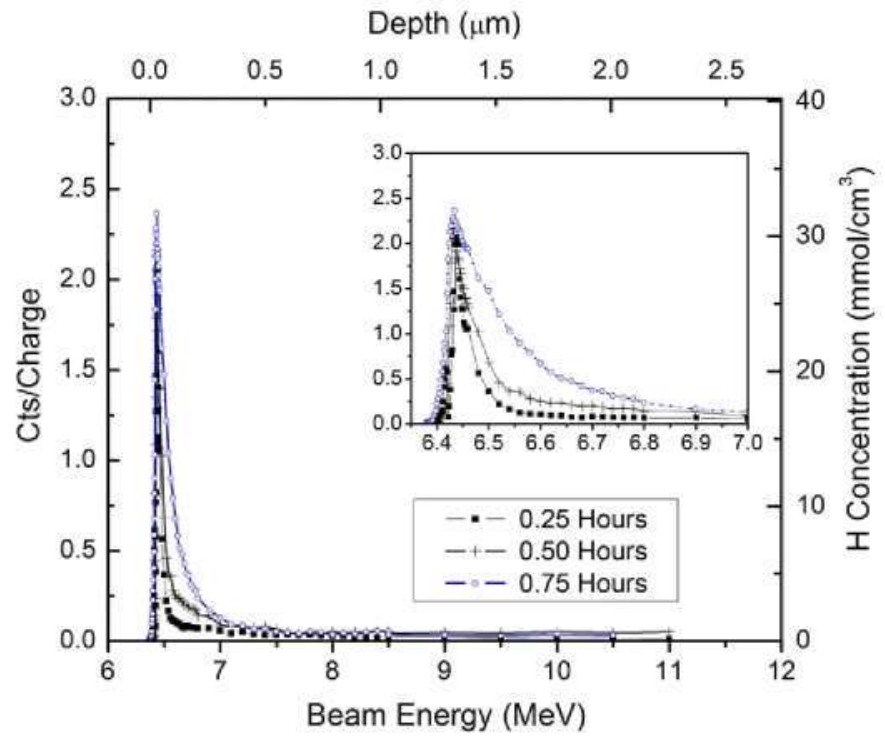

Fig. 1. Evolution of hydrogen depth profile for triclinic $\mathrm{C}_{3} \mathrm{~S}$ hydrated at $30^{\circ} \mathrm{C}$

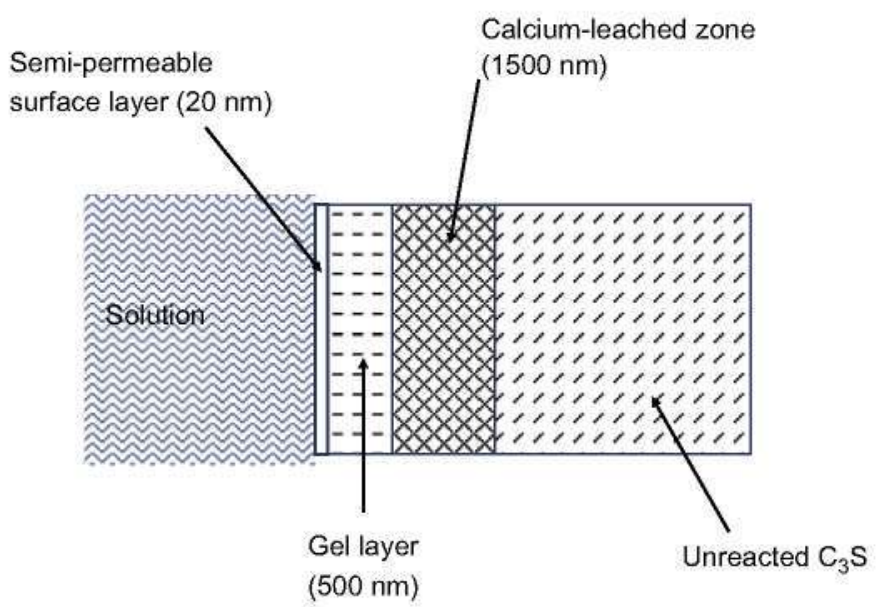

Fig. 2. Schematic diagram of surface layers based on $\mathrm{H}$ profiles.

layer, and it ruptures, releasing the trapped silica into the pore solution, where it reacts with the calcium ions to form the $\mathrm{C}-\mathrm{S}-\mathrm{H}$ gel. This breakdown of the surface layer marks the end of the induction period. This is easily recognized in the profiles by the absence of the Gaussian peak and a change in the shape of the diffusion region curve. It is possible to determine the time at the end of the induction period to a relative precision generally better than $5 \%$. Profiles for three different times are plotted in Fig. 1 to illustrate how the diffusion region grows while the Gaussian peak remains fixed.

In order to determine the shape of the profile more accurately and to optimize beam time usage, the energy increment of the beam is adjusted depending on the region of the profile. As shown in Fig. 1, at or near the surface the beam energy increases in fine $2 \mathrm{keV}$ steps to resolve thin surface layers. At 6.44 the step is increased to $10 \mathrm{keV}$. At $6.48 \mathrm{MeV}$ steps increased to $20 \mathrm{keV}$. For beam energies beyond $6.8 \mathrm{MeV}$, coarser steps (100-500 keV) are used as the profile typically changes more slowly in this region. The maximum beam energy was limited to $12 \mathrm{MeV}$ to avoid interference from the next higher energy resonance. In some cases, the analysis is stopped at lower maximum energies if the profile essentially drops to the baseline. The counting time is set to give a nominal total counts of 10,000 for the $4.44 \mathrm{MeV}$ peak for an uncertainty of $1 \%$. In practice, it has been found more convenient to stop counting when the beam has deposited $1.2 \mu \mathrm{C}$ of ${ }^{15} \mathrm{~N}$ charge. The beam current setting of $15 \mathrm{nA}$ represents a tradeoff between increased count rate and undesirable heating of the sample. This value of beam current yields a count rate of about $100 \mathrm{cps}$ in the $4.44 \mathrm{MeV}$ peak, and an individual $\gamma$-ray spectrum can thus be acquired in about a minute. Typically, about 80 spectra are collected for an individual profile. Including the time for sample mounting, vacuum pumping and beam adjustment for each energy increment, the total time required to acquire a complete depth profile is on the order of an hour and a half.

\subsection{Calibration}

As shown in Fig. 1, the NRRA coordinates are beam energy and counts per nanocoulomb of charge (typically a $2+$ charge state). These have to be converted to depth and $\mathrm{H}$ concentrations, respectively. For a given beam energy, $E_{B}$, the depth probed in the sample is approximately:

$x=\frac{E_{B}-E_{R}}{d E / d x}$ 


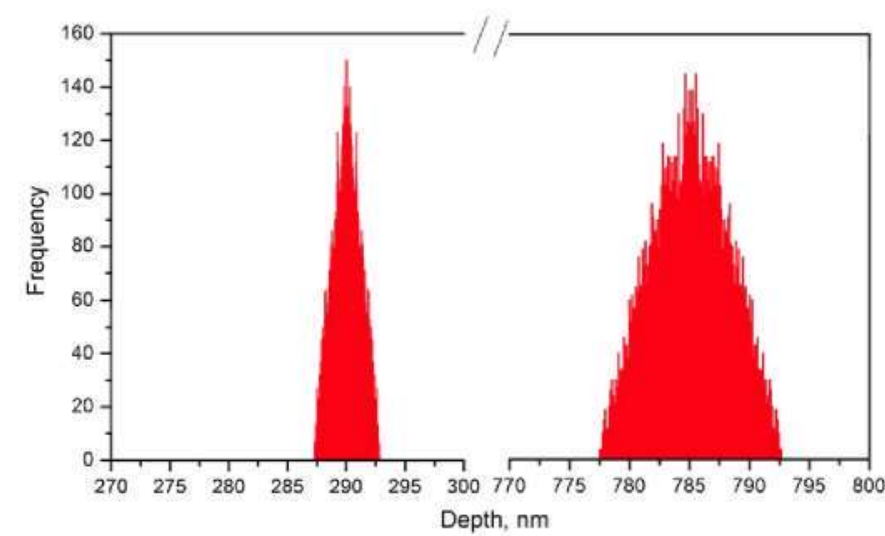

Fig. 3. Histograms of ${ }^{15} \mathrm{~N}$ TRIM simulations for $7 \mathrm{MeV}$ (left) and $8 \mathrm{MeV}$, (right). Note break in horizontal axis.

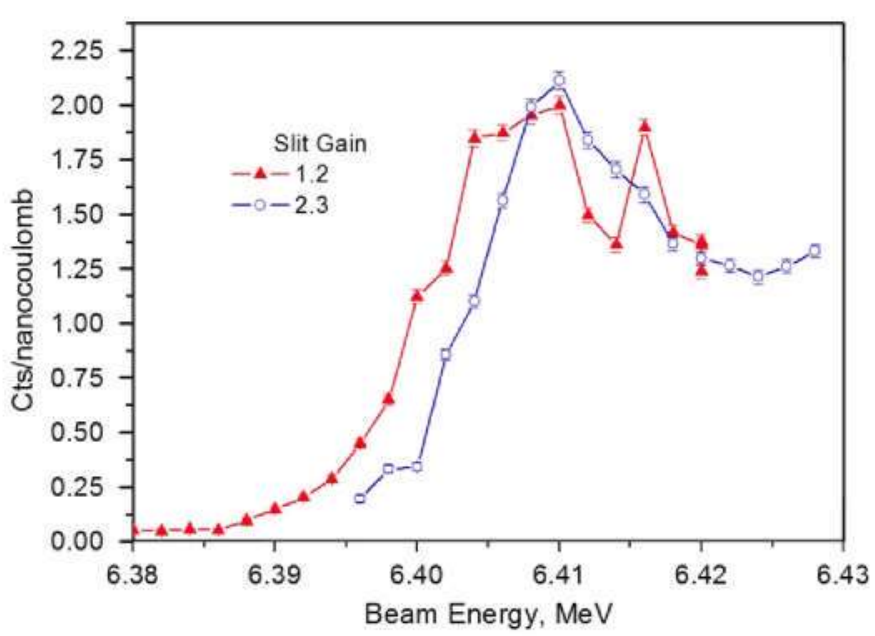

Fig. 4. H Depth profile on freshly cleaved mica surface used as a calibration standard. The two curves represent different tunings of the beam parameters. The triangles represent the original tuning and the open circles the result of the adjusting the slit gain.

where $E_{R}$ is the resonance energy, in this case $6.40 \mathrm{MeV}$, and $d E / d x$ is the average stopping power of the target material. The stopping power is a function of the number density and electron density of the various atoms in the sample. To determine the beam energy spread as a function of depth in the sample, a numerical simulation of the individual collisions is performed, usually with the Monte Carlo software TRIM (Ziegler et al., 1985). Fig. 3 shows some TRIM calculations for ${ }^{15} \mathrm{~N}$ in $\mathrm{C}_{3} \mathrm{~S}$. For $7 \mathrm{MeV}$ ions the resonance energy is reached at a depth of $290 \mathrm{~nm}$, and for the $8 \mathrm{MeV}$ ions at a depth of $785 \mathrm{~nm}$. The average stopping power is thus $2.02 \mathrm{keV} / \mathrm{nm}$. Fig. 3 also shows the effect of energy straggling with depth. The greater the number of atomic scattering events the particle undergoes before resonance capture the larger the width of the depth distribution around the nominal depth. Thus the energy resolution at the resonant energy for 7 and $8 \mathrm{MeV}$ incident beams has a fullwidth at half maximum of 19.3 and $64.3 \mathrm{keV}$, respectively. Note that these calculations assume an incident beam with an ideal delta-function distribution i.e. $\delta\left(E-E_{B}\right)$. The actual incident beam energy has a Gaussian distribution with a width of about $5 \mathrm{keV}$. Hence, the total beam energy width is this actual beam energy width combined in quadrature with the width for the energy straggling.

To determine the counts-to-H concentration conversion factor, measurements are made on a reference sample, which consists of a specimen of freshly cleaved muscovite mica. This has a very well established regular spacing of the hydrogen bearing layers (Abbott and Burnham, 1988) that provides a very uniform hydrogen concentration as a function of depth. Typical measurement results are shown in Fig. 4. At the thick target yield the counts reach a maximum or flat top that provides a very accurate measurement of the conversion factor from counts to $\mathrm{ppm}$ of $\mathrm{H}$.

In addition, the incident beam energy resolution can be estimated by the steepness of the slope of the curve as it rises to the thick target limit. Fig. 4 indicates how this resolution can be improved with suitable adjustment of the beam parameters. While the entire accelerator optics is optimized, this figure shows the effect of a small change in the gain used in the slit feedback system. This small part of the optics optimization improves the beam energy resolution on target by 1 or $2 \mathrm{keV}$.

The $\gamma$-ray counts to $\mathrm{H}$ concentration calibration is accomplished by using a calibrated reference sample. The conversion is given by (Rudolph et al., 1986)

$N_{H}=\frac{\left(\Gamma_{u} / \Gamma_{s t}\right) A_{s t} S_{c e m}}{1-\left(\Gamma_{u} / \Gamma_{s t}\right) A_{s t} S_{H}} N_{M}$

where $N_{H}$ is the hydrogen atomic concentration in the sample, $N_{M}$ is the atomic concentration of other atoms in the sample, $S_{c e m}$ is the stopping power of the cement matrix, $S_{H}$ is the stopping power of $H$, $A_{s t}$ is a factor that corrects for the difference in matrix composition between the standard and the sample, $\Gamma_{s t}$ is the area under the $4.44 \mathrm{MeV}$ peak for the standard, and $\Gamma_{u}$ the same area for the sample.

Finally, the depth profile measured by NRRA always starts at the current surface of the target which may not be the same as the original surface because of erosion of material during the hydration reaction. Thus some independent measurement method is required to determine the distance between the original and the current surface. Two methods have been used. One involves the deposition of a gold layer partially covering the sample surface prior to hydration. This provides a reference point for measuring the surface recession by profilometry. The other method implants a layer of xenon ions at a known depth prior to hydration. After hydration the current xenon layer depth is measured with Rutherford Backscattering (RBS). Xenon implantation has a much finer depth resolution, on the order of tens of nm (Perera and Doremus, 1991). However, preliminary experiments have shown that the implantation process may alter the reactivity of the target.

\section{Applications}

\subsection{Reactivity of cement phases}

One set of experiments investigated the development of the hydration profiles for different cementitious phases hydrated under fixed solution conditions, i.e. a $\mathrm{pH} 12.4$ solution saturated with respect to $\mathrm{CH}$. Both of the major gel-forming phases, tricalcium and dicalcium silicate, were studied as well as tricalcium aluminate which is also present. The first set of experiments was performed on triclinic $\mathrm{C}_{3} \mathrm{~S}$. Typical hydration profiles at $30^{\circ} \mathrm{C}$ are shown above in Fig. 1. The first set of experiments concerned the effect of temperature on the kinetics of the induction period. Isothermal hydration was conducted at three different temperatures: 10,20 or $30^{\circ} \mathrm{C}$. The result agreed very well with an Arrhenius-type behavior with an activation energy of $69 \pm 4 \mathrm{~kJ} / \mathrm{mol}$.

For comparison with $C_{3} S$, some depth profiles for $C_{3} A$ hydration are shown in Fig. 5. This phase is known to react very rapidly and can cause false setting of the concrete (Abbott and Burnham, 1988). For these hydration experiments, it was decided to try to slow the reaction by maintaining the solution at $10^{\circ} \mathrm{C}$. 


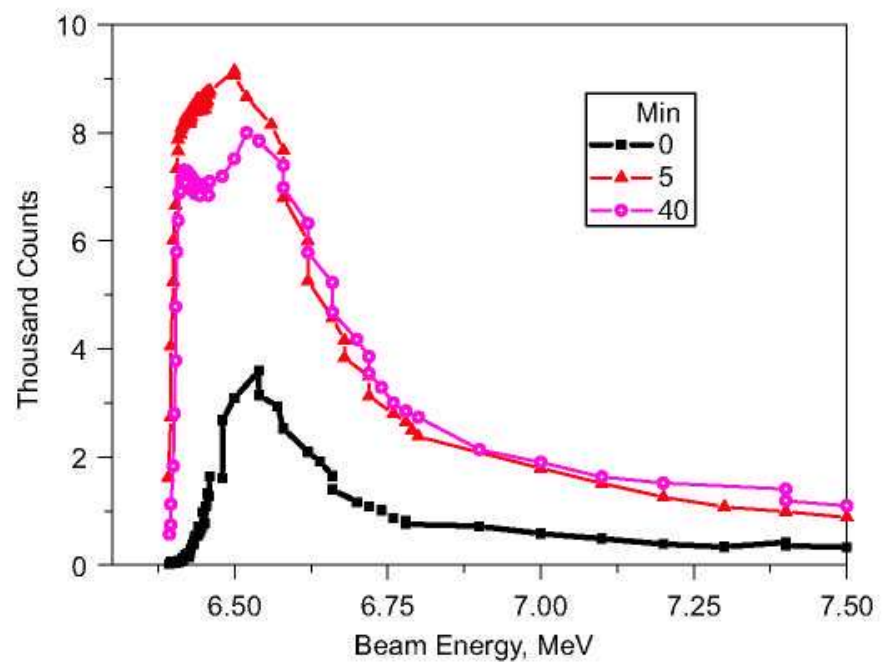

Fig. 5. H Depth profile in Tricalcium Aluminate.

Also some calcium sulfate was added to the solution to simulate the effect of gypsum normally found in Portland cement. The samples were removed from the solution at very short time intervals of 5 or $10 \mathrm{~min}$.

The resulting profiles (Fig. 5) are significantly different in shape from those for the $\mathrm{C}_{3} \mathrm{~S}$. Starting at the surface there is a very well defined vertical leading edge, which is interpreted as a layer of crystalline material. Given the nominal solution chemistry this would be a mixture of calcium hydroxide and ettringite (calcium aluminate sulfate hydrate). Next there is a notch, which is not evident at the early times, but tends to develop with time. This may represent the initial formation of an amorphous ettringite gel which subsequently crystallizes. Finally, there is a nonlinear gradient tailing off at higher energies which represents a zone of leaching and exchange between water and calcium.

With respect to hydration kinetics, the profile appears to be remarkably stationary with time. Fig. 5 shows the profiles at the earliest time of measurement, $5 \mathrm{~min}$, and at the last, $40 \mathrm{~min}$. The only apparent changes appear to be the development of the notch and the reduced height of the main peak. Unlike the $C_{3} S$ profile, the gradient region does not continue to grow deeper into the substrate. In other words, in the first $5 \mathrm{~min}$, the diffusion of water reaches a critical depth and then stops as the reaction at the surface forms a crystalline layer that acts as an impermeable barrier which completely stops further diffusion and reaction.

\subsection{Accelerators and retarders}

As noted above, there are a number of chemicals that can accelerate or retard the hydration process (Abbott and Burnham, 1988). They can be simple inorganic salts like calcium chloride or complex organic compounds, often involving carboxylates. For example, lignosulfonates, often used as retarders, are waste products from the wood pulp industry and consist of threedimensional random polymers with molecular weights up to 50,000 . They also contain various other organic compounds such as sugars and gluconates that also act as retarders. At present, mechanisms through which acceleration and retardation occurs are not completely understood. Some theories maintain that it involves absorption on surfaces, others that it involves effects on solubility of various chemical species.

As a preliminary step in investigating these effects some hydration experiments were carried out on $\mathrm{C}_{3} \mathrm{~S}$ pellets using either a classic accelerator, calcium chloride, or a retarder,

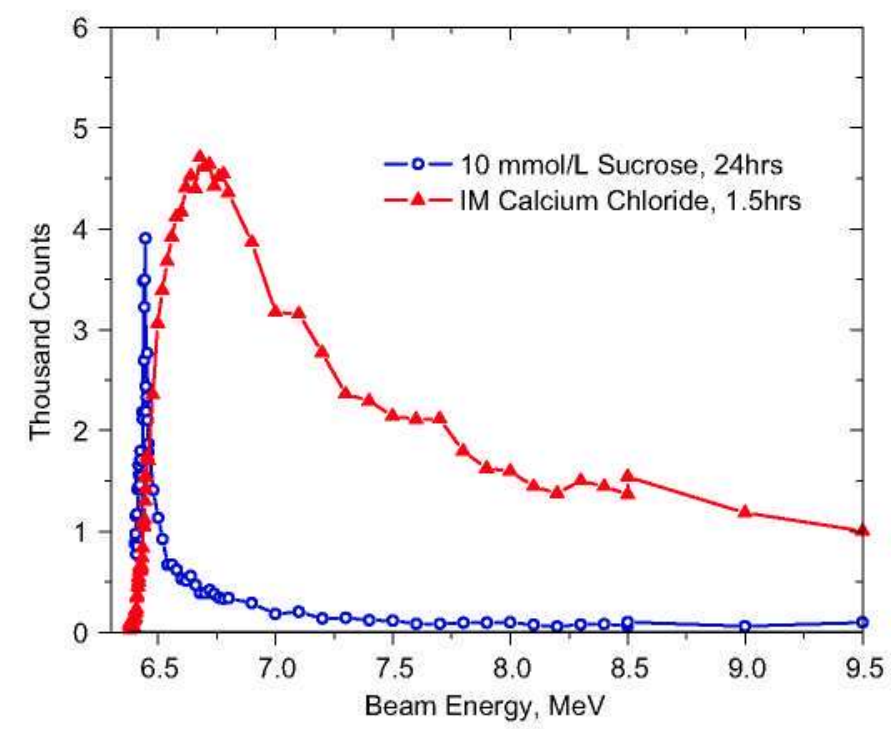

Fig. 6. H Depth profiles for set modifiers.

sucrose. A comparison of the profiles is given in Fig. 6. Both samples were hydrated at $30^{\circ} \mathrm{C}$. For the calcium chloride solution, at $1.5 \mathrm{~h}$, the surface layer has already broken down. In contrast, for the sucrose at $24 \mathrm{~h}$, the longest time interval reached in these experiments, the surface layer is still present, and the diffusion region has barely started to develop.

Current research on retarders is focused on sodium gluconate, which is a major ingredient in commercially available retarders. The initial set of experiments has shown that adding as little as $0.013 \%$ by weight of sodium gluconate to the solution significantly reduces the rate of hydration. Increasing the dosage to $0.018 \%$ slows the hydration by an additional factor of 2.7 .

\section{Conclusions}

The spatial sensitivity of the ${ }^{1} \mathrm{H}\left({ }^{15} \mathrm{~N}, \alpha, \gamma\right){ }^{12} \mathrm{C}$ reaction provides a novel and unique method to investigate the physical and chemical processes involved in the hydration of various components of Portland cement at the nanometer scale. This has provided new insights into the mechanisms that control the setting and curing of concrete and has helped to resolve some longstanding controversies. For the calcium silicate phases, there is generally the development of a semi-permeable surface layer that controls the hydration rate of reaction during the induction period. The length of the induction period shows a classic Arrhenius-type dependence on temperature.

Tricalcium aluminate shows a very different spatial pattern of hydration. There is no semi-permeable surface layer. Instead a crystalline layer develops almost immediately, and apparently halts further reaction with water.

Initial experiments with accelerators and retarders have shown that they significantly affect the development of the hydration profile, either by changing the permeability of the surface layer or the diffusion coefficients in the substrate. For a commercially available retarder like sodium gluconate, the rate of hydration is strongly dependent on the dosage.

\section{Acknowledgement}

The authors are indebted to the support of the National Science Foundation under contract CMS-0600532 that made this research possible. 


\section{References}

Abbott, R.N., Bumham, C.W., 1988. Polytypism in micas: a polyhedral approach to energy calculations. American Mineralogist 73, 105-118.

Borucki, l., Becker, H.W., Gorris, F., Kubsky, S., Schulte, W.H., Rolfs, C., 1999 Hydrogen doppler spectroscopy using ${ }^{15} \mathrm{~N}$ ions. European Journal of Physics A 5. 327-336.

Henderson, E., Bailey. J.E., 1993. The compositional and molecular character of the calcium silicate hydrates formed in the paste hydration of $3 \mathrm{CaOSiO}_{2}$. Journal of Materials science 28, 3681-3691.

Lanford, W.A., 1995. Nuclear Reactions for Hydrogen Analysis. In: Tesmer, J., Nastasi, M. (Eds.), Handbook of Modem lon Beam Materials Analysis. Materials Research Society. Pittsburgh. pp. 193.

livingston, R.A., Schweitzer, J.S., Rolfs, C., Becker, H.-W., Kubsky, S., 2001 Characterization of the Induction Period in Tricalcium Silicate Hydration by Nuclear Resonance Reaction Analysis. Journal of Materials Research 16 (3). $687-693$.

Menetrier, D., Jawed. I., Sun. T.5., Skalny. J., 1979. ESCA and SEM Studies of Early $\mathrm{C}_{3}$ S Hydration. Cement and Concrete Research 9, 473.

Mereditl, P., Donald. A.M., luke, K., 1995. Pre-induction and induction hydration of tricalcium silicate: An environmental scanning electron microscope study. Journal of Materials Science 30.1921-1930.

Mitchell, L.D., Prica, M., Birchall, J.D., 1996. Aspects of Portland cement hydration studied using atomic force microscopy. Journal of Materials Science 31, 4207-4212.

Perera, G., Doremus, R.H., 1991. Dissolution rates of commercial soda-lime and pyrex borosilicate glasses: influence of solution $\mathrm{pH}$. Joumal of the American Celamic Society $74(7), 1554-1558$.

Rudolph, W., Bauer, C., Brankolf, K., Grambole, D., Grotzschel, R., Heiser, C. Herrmann, F., 1986. Plastic foils as primary hydrogen standards for nuclear reaction analysis. Nuclear Instruments and Methods in Pliysics Research B 15. 508-511.

Schweitzer, J.S., Livingston, R.A., Rolfs, C., Becker, H.-W., Kubsky, S., 2001. Study of Cement Chemistry with Nuclear Resonant Reaction Analysis. Applications of Accelerators in Research and Industry, in: Duggan, J. L., Morgan, l. L. Proceedings of the Sixteenth International Conference. Denton. Texas, American lnstitute of Plyysics. 576, 1077-1080.

Schweitzer, J.S., Livingston, R.A., Rolfs, C., Becker, H.-W., Kubsky, S., 2003. Ion beam analysis of the hydration of tricalcium silicate. Nuclear Instruments and Methods in Physics Research B 207 (1), 80-84.

schweitzer, J.5., livingston, R.A., Rolf5, C., Becker, H.-W., Kubsky, S., Spillane, T., Castellote, M., Garcia de Viedma, P., 2005. Nanoscale studies of cement chemistry with $15 \mathrm{~N}$ resonance reaction analysis. Nuclear Instruments and Methods in Pliysics Research B 241, 441-445.

Scrivener, K, 1987. The Microstructure of Anhydrous Cement and its Elfect on Hydration. In: Struble, L.]., Brown, P.W. (Eds.), Microstructural Development During Hydration of Cement, Vol. 85. Materials Research Society. Pittsburgh. pp. $39-46$

Taylor, H.F.W., 1997. Cement Chemistry, second ed Thomas Telford, London.

Viehland, D., li. J.-F., Yuan, l.,-J., Xu. Z., 1996. Mesostructure of calcium silicate hydrate $(c-s-h)$ gels in Portland cement paste: short-range ordering, nanociystallinity, and local compositional order. Journal of the American Ceramic Society 79 (7), 1731-1744.

Wüstenbecker, 5., Becker, H.W., Rolfs, C., Trautvetter, H.P., Brand, K., Mitchell, G.E., schweitzer, J.S., 1987. Technical developments for ion beams with high energy resolution. Nuclear lnstruments and Methods A 256, 9-22.

Young. J.F., 2005. Tricalcium Silicate Hydration: A Historical overview, In: Young. J.F., Skalny. J. (Eds.), Materials Science of Concrete VII. The American Ceramic Society. Westerville, $0 \mathrm{H}, \mathrm{pp}, 101-118$.

Ziegler, J.F., Biersack, J.P., Littmark, U., 1985. The Stopping and Range of lons in Solids. Pergamon Press, New York. 